\title{
The electronic structure of ionic liquids based on the TFSI anion: a gas phase UPS and DFT study
}

\author{
Ivar Kuusik $^{\mathrm{a}}$, Mati Kook ${ }^{\mathrm{a}}$, Rainer Pärna ${ }^{\mathrm{a}, \mathrm{b}}$, Antti Kivimäki ${ }^{\mathrm{c}, \mathrm{b}}$, Tanel Käämbre ${ }^{\mathrm{a}}$, Liis \\ Reisberga, $^{\mathrm{a}, \mathrm{b}}$, Arvo Kikas ${ }^{\mathrm{a}}$, Vambola Kisand $^{\mathrm{a}}$ \\ ${ }^{\text {a }}$ Institute of Physics, University of Tartu, W. Ostwaldi 1, EE-50411 Tartu, Estonia \\ ${ }^{\mathrm{b}}$ MAX IV Laboratory, Lund University, Fotongatan 2, 22594 Lund, Sweden \\ ${ }^{c}$ Nano and Molecular Systems Research Unit, University of Oulu, P.O. Box 3000, Oulu, \\ Finland
}

\begin{abstract}

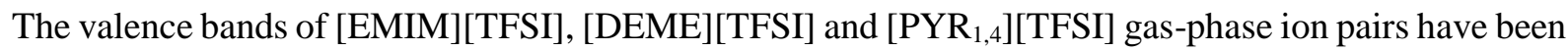
investigated using ultraviolet photoelectron spectroscopy (UPS). The photoelectron spectra are interpreted by using several density functional and $a b$ initio calculation methods. Although the experimental vapor phase spectra are similar, the different calculation methods make different predictions about the HOMO molecular state of the ion-pairs of the ionic liquids. The HOMO state of the [DEME][TFSI] ion-pair is due to the TFSI anion, while in [EMIM][TFSI] it is due to the EMIM cation. However, it is difficult to make conclusive assignments for the $\left[\mathrm{PYR}_{1,4}\right][\mathrm{TFSI}]$ ionic liquid. All calculation methods predict the LUMO to be of cationic origin in all the studied ion-pairs.
\end{abstract}

\section{Introduction}

Ionic liquids (ILs) are generally defined as molten organic salts with a melting point below $100{ }^{\circ} \mathrm{C}$. These compounds have attracted high interest because of their uncommon physicochemical properties such as low melting temperatures, excellent solvation ability, relatively high thermal stability, low vapor pressure, non-flammability, high electrochemical stability etc. $[1,2]$. Synthesizing a vast number of different ILs is possible with the selection of many available anions and cations. One important application for ionic liquids is as an electrolyte in electrochemical double-layer capacitors (EDLCs), or supercapacitors [3]. In that application, the ionic conductivity and the width of the electrochemical stability window are the most important properties of ILs. 
The three ILs under investigation in the present work are: [PYR 1,4$][T F S I]$ (1-Butyl-1methylpyrrolidinium bis(trifluoromethylsulfonyl)imide), [DEME][TFSI] (diethylmethyl (2methoxyethyl)ammonium bis(trifluoromethylsulfonyl)imide) and [EMIM][TFSI] (1-Ethyl-3methylimidazolium bis(trifluoromethylsulfonyl)imide) (see structures in Figure 1.). We use the simplest notation for the ILs, which is based on the cations and anions: [CATION][ANION]. [PYR 1,4$][$ TFSI] and [DEME][TFSI] are promising ILs for EDLCs [4, 5], [EMIM][TFSI] has already shown good properties in EDLC, including low cost and high cyclability.

In the first approximation, the electrochemical stability window (EW) of ILs is determined by the HOMO and LUMO energies of the ion-pairs they consist of [6-8]. The upper end of the electrochemical window is usually limited by the oxidation of the anion, and the lower end of the electrochemical window is determined by the reduction of the cation [9]. Ilawe et al. also claimed that the HOMOLUMO gap of the ion-pairs is an indicator of IL stability [10]. Lian et al. claimed that the cathodic and anodic limits can be reasonably estimated from the relation $\mathrm{V}_{\mathrm{cl}}=\max \left(\mathrm{LUMO}_{\text {cation }}, \mathrm{LUMO}_{\text {anion }}\right)$, $\mathrm{V}_{\mathrm{al}}=\min \left(\mathrm{HOMO}_{\text {cation }}, \mathrm{HOMO}_{\text {anion }}\right)$, where the energies of the individual cations and anions (in vacuum) are used [11]. Very recently, they claimed that this energy gap approximation to the EW calculation has already been verified [12]. Using the $\omega \mathrm{B} 97 \mathrm{X}-\mathrm{D}$ functional for the calculations, they calculated the EWs of [EMIM][TFSI] and [PYR 1,4$][$ TFSI] to be about $3.6 \mathrm{~V}$ and $4.2 \mathrm{~V}$ respectively. However, Peljo and Girault claim that these kinds of ion-pair approximations to the EW should be discarded completely [13]. We concur with Peljo and Girault in that the HOMO, LUMO approximation to the EW may fail, but believe that to make comparisons between similar ILs, the approximation may give some insights. Due to the high importance, we will try to deduce the origin of the HOMO and LUMO states in the vapor of these ionic liquids.

Using similar experimental methods we have previously investigated the valence band photoelectron spectra of simple molecules like $\mathrm{CsCl}$ [14] and rubidium halides [15]. Gas phase experiments on ILs are possible, despite the fact that ionic liquids have very low vapor pressures $[2,16]$. We have previously used UPS spectroscopy and ab initio calculations for the study of [EMIM] $\left[\mathrm{BF}_{4}\right]$ in both vapor and liquid phase [17, 18] and for trimesic acid molecules [19].

To the best of our knowledge, this is the first presentation of the vapor phase UPS spectra of two TFSI based ionic liquids: [DEME][TFSI] and [PYR $\left.{ }_{1,4}\right][$ TFSI]. Liquid phase UPS spectra of these ILs are also not yet available, but there are some theoretical works about [PYR 1,4$]$ [TFSI] [20-22]. The UPS spectrum of gas-phase [EMIM][TFSI] has already been published by Strasser et al. [23]. Their and our independently measured spectra are highly similar, thus confirming their correctness. The UPS spectrum of liquid [EMIM][TFSI] has already been published by several groups [24-29] The liquid and gas-phase 
spectra of [EMIM][TFSI] are qualitatively highly similar. However, there is quite a substantial shift in binding energy between the corresponding features in the liquid and gas phase spectra.

\section{Experimental}

For the gas-phase experiments [EMIM][TFSI] (purity $\geq 98 \%$ ), [PYR 1,4$][\mathrm{TFSI}$ (purity $\geq 98.0 \%$ ) and [DEME][TFSI] (purity $\geq 98.5 \%$ ) were purchased from Sigma Aldrich. Gas-phase experiments were carried out by evaporating the liquid from a quartz crucible of an effusion cell (MBE Komponeten NTEZ-40-10-22). After inserting the IL into the effusion cell, heating of IL at $80{ }^{\circ} \mathrm{C}$ for several hours was performed to remove residual water from the chemical. However, there are still traces of water vapor visible in the spectra of $\left[\mathrm{PYR}_{1,4}\right][\mathrm{TFSI}]$ and [DEME][TFSI] (the peak at $12.6 \mathrm{eV}$, between the peaks $\mathrm{C}$ and D). The ILs themselves were evaporated at $220-240{ }^{\circ} \mathrm{C}$.

The UPS measurements of gas-phase IL-s were carried out at the new FinEstBeAMS beamline of the MAX-IV $1.5 \mathrm{GeV}$ storage ring (Lund, Sweden) [30, 31]. The beamline covers the excitation photon energy range from $4.5 \mathrm{eV}$ to about $1300 \mathrm{eV}$. The beamline is equipped with a collimating grazing incidence plane grating monochromator and toroidal focusing mirrors. The $92 \mathrm{~mm} / 1$ grating was used in the present work. UPS spectra were obtained using an electron energy analyzer (SCIENTA R-4000) in the fixed analyzer transmission mode with the pass energy of $20 \mathrm{eV}$ (low pass regime). UPS measurements were carried out in $10^{-7}$ mbar pressure range with a liquid nitrogen cooled cold trap. UPS spectra were measured with an excitation energy $40 \mathrm{eV}$ ([EMIM and PYR , $\left._{1,4}\right]$ [TFSI]) or $50 \mathrm{eV}$ ([DEME][TFSI]). Binding energies were calibrated to the $\mathrm{Ar} 3 \mathrm{p}_{3 / 2}(15.76 \mathrm{eV})$ photoelectron lines [32]. Argon was intentionally dosed into the analysis chamber for energy calibration purpose during some measurements. The Ar $2 p$ photoelectron line was substracted from the raw [EMIM][TFSI] spectrum.

DFT and $a b$ initio Møller-Plesset perturbation theory (MP2) calculations were performed using Spartan 14 software [33]. The standard BLYP functional and the hybrid functionals B3LYP, M06 and $\omega$ B97XD [34] were used. The gaussian basis set $6-311++G^{* *}$ (basis set with d,p-polarization and diffuse functions) was used for the BLYP, B3LYP and M06 functionals. Due to the high computational cost of the 6-311++ $\mathrm{G}^{* *}$ basis set, the gaussian basis set 6-311 has been used for the MP2 calculations. In case of the $\omega \mathrm{B} 97 \mathrm{X}-\mathrm{D}$ functional, the $6-311++\mathrm{G}^{* *}$ basis set has been used for [EMIM][TFSI] and [PYR $\left.{ }_{1,4}\right][T F S I]$ ion-pairs and the $6-311+\mathrm{G}^{* *}$ basis set has been used for the [DEME][TFSI]. The 6$311++\mathrm{G}^{* *}$ and the $6-311+\mathrm{G}^{* *}$ basis sets are expected to produce almost identical results. Many different ion-pair conformers were manually constructed. Each geometry was optimized (relaxed) for lowest energy. For example, 17 different conformers were studied for [DEME][TFSI]. The density of states (DOS) type spectra shown in Fig. 2 were obtained by convoluting the calculated discrete states 
with a Gaussian function ( $0.7 \mathrm{eV}$ full width at half maximum) under the assumption that the electron emission intensities from each orbital are the same. In case of the MP2 calculations, the energy scale was compressed to $85 \%$. The calculated DOS was shifted to align the peaks and make comparison between the experimental spectra and the simulated DOS easier. The shifts used were $0.5 \mathrm{eV}, 0.3 \mathrm{eV}$ and $2.0 \mathrm{eV}$, for the MP2, $\omega$ B97X-D and M06 functionals, respectively.

\section{Results and Discussion}

The UPS spectra of [EMIM][TFSI], [DEME][TFSI] and [PYR 1,4] $_{\text {[TFSI] }}$ are shown in Fig. 2. 12 different photoelectron peaks labeled A to J were distinguished by fitting the experimental UPS spectra (see Fig. 2). These peaks do not represent distinct states, but are used just for labelling and referring to the experimental spectra. When the comparison between our vapor phase [EMIM][TFSI] UPS spectrum and the liquid phase XPS spectrum of Ikari et al. or Kurisaki et al. is made, the biggest difference is the lack of the inelastic scattering background and less broadening in our gas-phase spectrum [24, 25].
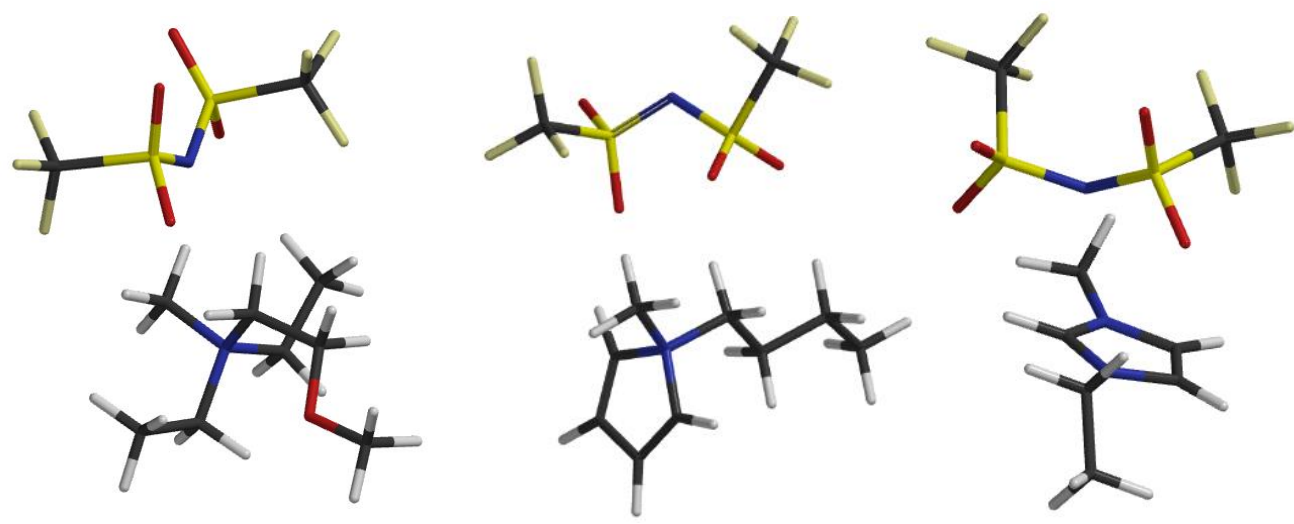

Figure 1. From left to right: predicted structures of [DEME][TFSI] , [PYR 1,4$][\mathrm{TFSI}]$ and [EMIM][TFSI] gas-phase ion pairs. The different bonds are shown using different colors: gray (hydrogen), black (carbon), blue (nitrogen), red (oxygen), green (fluorine) and yellow (sulfur). The structures have been optimized using the hybrid $\omega$ B97X-D functional.

There are three clearly separable regions in all spectra: peaks A-C, peaks E and F and peak $\mathrm{H}$. The peaks $\mathrm{E}$ and $\mathrm{F}$ are the strongest peaks in all cases. The intensity of peak $\mathrm{F}$ (compared to peak $\mathrm{E}$ ) varies somewhat between the three ILs. However, the biggest difference between the spectra of the three IL vapors is in the intensity of the peak D. It is strong in [PYR $\left.{ }_{1,4}\right][\mathrm{TFSI}]$ and weak in [EMIM][TFSI], when compared to [DEME][TFSI], which is in-between the two. The intensity ratio between peaks B and C also varies between the three ILs. This variation is even stronger in the theoretically simulated DOS type 
spectra. The peak $\mathrm{C}$ is stronger than the peak $\mathrm{B}$ in the spectrum of [EMIM][TFSI], while the opposite is true in $[\mathrm{DEME}][\mathrm{TFSI}]$. In case of [PYR $\left.\mathrm{P}_{1,4}\right][\mathrm{TFSI}]$, the peaks are almost of equal intensity.

However, when discussing intensities, it is important to remember that the intensity of photoemission does not depend on the density of states only [35] but also on the experimental geometry, electron analyzer transmission function, incident photon energy and photoemission cross sections among other parameters. For example, the peaks I and $\mathrm{J}$ are more intense when higher excitation photon energy is used $[24,25]$.

Next the calculation results will be discussed. The choice of a calculation method for ionic liquid ionpairs is not trivial $[17,36]$. Although wavefunction based methods can be highly accurate and are mostly parameter-free, they tend to be computationally too expensive for larger systems. It would be beneficial to employ Kohn-Sham density functional theory calculation methods.

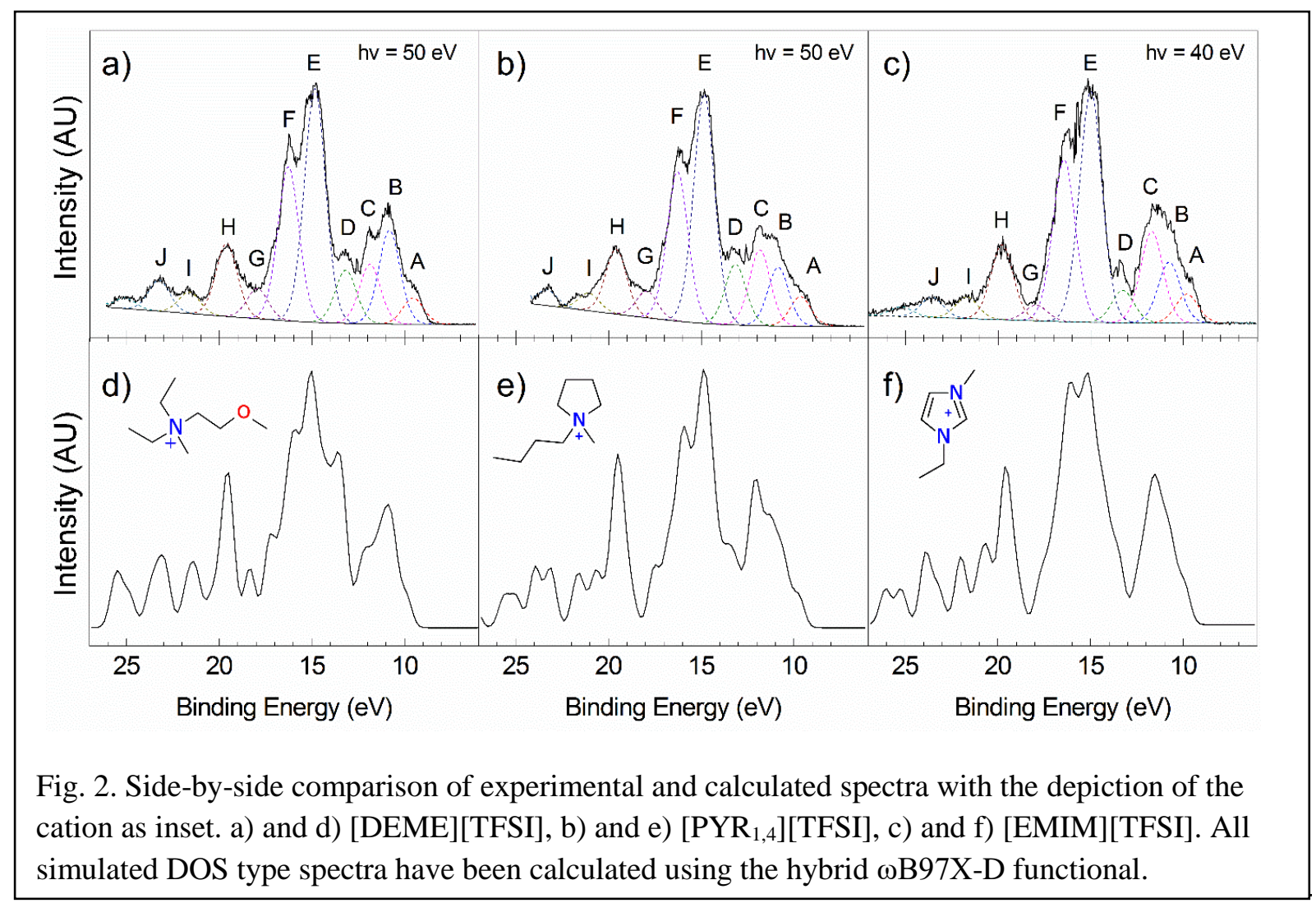

The MP2 method is a proven ab initio calculation method. In the case of [EMIM] $\left[\mathrm{BF}_{4}\right]$ ion-pairs, only the MP2 calculation described the HOMO level correctly [17]. Similarly, in the case of $[\mathrm{EMIM}]\left[\mathrm{B}(\mathrm{CN})_{4}\right] \mathrm{IL}$, again only the MP2 ion-pair approximation was able to describe the top of the valence band [37] . However, in case of the ionic liquids with the TFSI anions studied here, the MP2 calculated DOS of the [EMIM or PYR $\left.{ }_{1,4}\right][$ TFSI] ILs has a too big separation of the HOMO level from the other orbitals. Like in many other cases, where calculations based on the Hartree-Fock method are used, the energy scale of the MP2 calculation needs to be compressed by about $15 \%$ to match the 
experiment [38]. The DOS calculated by the MP2 method was qualitatively correct, but there were also some other small disagreements with experimental spectra.

Yildirim et al. claimed that the hybrid functionals likely predict a more accurate picture for the [EMIM] $\left[\mathrm{BF}_{4}\right]$ ion-pair than the standard GGA functionals. Indeed, hybrid functionals tend to perform better than the non-hybrid ones, but are also computationally more expensive. For example, the nonhybrid functional BLYP offers poor description of the electronic structure of [DEME][TFSI]. The hybrid functionals B3LYP, M06 and $\omega$ B97X-D were evaluated. The B3LYP functional is clearly inferior to the M06 and $\omega$ B97X-D functionals. Similarly to its nonhybrid cousin (BLYP), the energy scale does not match the experiment and there are some serious shortcomings in the description of the [DEME][TFSI] UPS spectrum.

The M06 and $\omega$ B97X-D functionals, however, are similar and offer overall excellent description of the

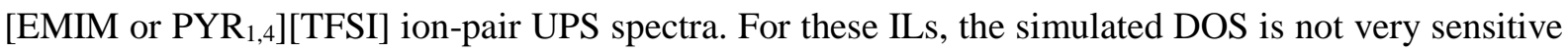
to the underlying structure as different conformers have similar simulated DOS. In addition, there is no need for energy scale adjustment other than a constant shift. This is surprising, since in most other cases, the energy scale would benefit from compressing in the case of ion-pair calculations [17, 18] or stretching when bulk calculations are performed [37] . The M06 functional needed a $2.0 \mathrm{eV}$ shift and the $\omega \mathrm{B} 97 \mathrm{X}-\mathrm{D}$ functional only needed a $0.3 \mathrm{eV}$ shift to match the experiment. This excellent performance of the $\omega \mathrm{B} 97 \mathrm{X}-\mathrm{D}$ functional is not surprising, since it is close to the absolute best in the benchmark studies [39]. Ilawe et al. also found that the $\omega \mathrm{B} 97 \mathrm{X}-\mathrm{D}$ functional is superior to M06-2X and B3LYP [10]. Lian et al. also used the $\omega \mathrm{B} 97 \mathrm{X}-\mathrm{D}$ functional and claimed that it is quantitatively accurate for predicting the electronic properties of individual ions in vacuum [11].

The description of the [DEME][TFSI] ion-pair is the most challenging for theory. For example, the B3LYP functional has the intensity of the main peaks ( $E$ and F) reversed. Also, the low binding energy part of the spectrum (peaks A-D) is very sensitive on the ion-pair structure. The structure shown in Fig. 1 represents the conformer whose calculated electronic structure agrees best with the experimental UPS spectrum. Still, the intensity of the peak D is substantially overestimated. It is also important to point out that neither the experimental nor the theoretical spectra may represent the lowest energy conformer, since the evaporation temperature was about 500K. Reinmöller et al. showed that in the case of [EMIM][TFSI], the different conformers have minor influence on the reconstructed XPS valence band spectrum and the HOMO.

According to Yildirim et al., the HOMO of [PYR $\left.{ }_{1,4}\right][\mathrm{TFSI}]$ is dominated by the TFSI anion, while the LUMO is due to the $\mathrm{PYR}_{1,4}$ cation. Indeed, our $\omega \mathrm{B} 97 \mathrm{X}-\mathrm{D}$ calculation confirms this result. The largest contribution to the HOMO level comes from the $\mathrm{N}$ and $\mathrm{O}$ atoms of the TFSI anion, which is also exactly what Yildirim et al. found for [PYR 1,4$][\mathrm{TFSI}][20]$. 
Our M06 ion-pair calculation predicts a HOMO-LUMO gap of $4.88 \mathrm{eV}, 6.00 \mathrm{eV}$ and $6.19 \mathrm{eV}$ for $\left[\mathrm{PYR}_{1,4}\right.$ , DEME, EMIM][TFSI], respectively. Yildirim et al. [20] calculated the HOMO-LUMO gap of $\left[\mathrm{PYR}_{1,4}\right][\mathrm{TFSI}]$ to be about $6.03 \mathrm{eV}$ using the hybrid HSE06 functional. This gap estimate is probably closer to reality than our M06 estimate, since for the [EMIM] $\left[\mathrm{BF}_{4}\right] \mathrm{IL}$, the HSE06 functional predicts a gap very close to the experimental value, while the M06 functional underestimates it somewhat $[17,20]$.

As stated above, it is possible to relate the HOMO-LUMO gaps of the IL ion-pairs to their liquid phase electrochemical windows [11]. From our calculated HOMO-LUMO gaps, it would seem that EMIM is the most electrochemically stable cation, followed by DEME and PYR $\mathrm{R}_{1,4}$. However, Mousavi et al. pointed out that aromatic cations generally have lower LUMO energy levels, which makes them more susceptible to reduction [3] . This would imply on the contrary, that [DEME][TFSI] should be the most electrochemically stable. Indeed, experimentally DEME is somewhat more stable than PYR $\mathrm{R}_{1,4}$ (by about $0.3 \mathrm{~V},[3])$ and they are both significantly more stable than EMIM (by about $1 \mathrm{~V}$ or more) [3]. Haskins et al. claimed that $\left[\mathrm{PYR}_{1,4}\right][\mathrm{TFSI}]$ has a $1 \mathrm{~V}$ wider EW than [EMIM][BF 4 [22]. Matsumoto et al. have also demonstrated high cationic stabilities of about $-3.4 \mathrm{~V}$ vs $\mathrm{Fc} / \mathrm{Fc}+$ for $\left[\mathrm{N}_{1113}\right][\mathrm{TFSI}]$ and $\left[\mathrm{N}_{5555}\right][\mathrm{TFSI}]$, which are similar ILs to [DEME][TFSI].

Since the anion is the same for all ILs studied here, the differences in the widths of the electrochemical windows must be due to the cations. The shoulder to the A peak extends to about $8.7 \mathrm{eV}$ binding energy in all three spectra. However, some of the [PYR 1,4$][\mathrm{TFSI}]$ UPS spectra have an unexplainable low intensity low binding energy tail that extends by about $1 \mathrm{eV}$ to even lower energies than the main shoulder to the peak A (i.e it extends to $7.7 \mathrm{eV}$ ). It will be ignored in the following discussion and further study on the origin of this tail will be conducted in the near future. Therefore, all three ILs have a similar low binding energy region (the shoulder to the peak A), thus suggesting initially that the HOMO level of the ILs is due to the TFSI anion.

To gain further insights into the nature of the HOMO state, results from the theoretical calculations need to be discussed. The failure of DFT to describe the HOMO states of [EMIM] $\left[\mathrm{BF}_{4}\right.$ or $\left.\mathrm{B}(\mathrm{CN})_{4}\right][17,18$, 37] (and possibly the similar $\mathrm{PYR}_{1,4}$ cations) is already known. Most DFT functionals predict the HOMO to be associated with the anion, while in the case of [EMIM][TFSI] (and possibly $\left[\mathrm{PYR}_{1,4}\right][\mathrm{TFSI}]$ ) ion-pairs, it is due to the $\pi$-states of the (aromatic) imidazolium (or pyrrolidinium) ring. Both the $\omega \mathrm{B} 97 \mathrm{X}-\mathrm{D}$ and M06 functionals predict the HOMO of [PYR $\left.{ }_{1,4}\right][\mathrm{TFSI}]$ to be localized on the anion. However, for [EMIM][TFSI] they predict the HOMO to be associated with both the cation and the anion. This so-called mixed assignment is very interesting and it puts the hybrid M06 and $\omega \mathrm{B} 97 \mathrm{X}-$ D functionals in-between the MP2 (see discussion below) and the standard DFT functionals. For example, the standard DFT functional BLYP predicts the HOMO level of [EMIM][TFSI] to be localized on the anion. 
In contrast, the MP2 calculation predicts the HOMO of [PYR ${ }_{1,4}$ or EMIM][TFSI] ion-pairs to be due to the aromatic ring of the cation. The MP2 calculation predicts the HOMO state of [DEME][TFSI] to be associated with the TFSI anion. Therefore, in case of [DEME][TFSI], all calculation methods predict the HOMO to be localized on the anion. Similarly to the DFT functionals, the MP2 calculation also predicts the LUMO to be of cationic origin in all ([DEME or EMIM or PYR $\left.{ }_{1,4}\right][\mathrm{TFSI}]$ ) ion-pairs.

Finally, it is important to point out the ion-pair approximation when comparing the electronic structure of ion-pairs and the liquid phase. The ion-pair approximation assumes that the electronic structure of the ionic liquid is similar to the ion-pairs it consists of. The ion-pair approximation seems to hold for [EMIM][TFSI] [29] but may not be valid for $\left[\mathrm{PYR}_{1,4}\right.$ or DEME][TFSI], a situation similar to [EMIM] $\left[\mathrm{BF}_{4}\right.$ or $\left.\mathrm{B}(\mathrm{CN})_{4}\right][18,37]$. Therefore care should be taken when extrapolating results from these gas phase measurements and calculations to the liquid phase.

\section{Conclusions}

Gas-phase ion-pairs of [EMIM][TFSI], [DEME][TFSI] and [PYR $\left.{ }_{1,4}\right][\mathrm{TFSI}]$ were investigated using valence band photoemission. Although their electronic spectra are similar in their overall shape, the spectral region around $12 \mathrm{eV}$ (peaks B-D) was sufficiently different. The DFT calculation using the $\omega \mathrm{B} 97 \mathrm{X}-\mathrm{D}$ functional was able to reproduce most of the spectral features. All calculation methods also predict the LUMO to be of cationic origin in all ([DEME|EMIM|PYR $\left.{ }_{1,4}\right][\mathrm{TFSI}]$ ) ion-pairs. However the HOMO level of all three ILs needs to be discussed separately.

In the case of [DEME][TFSI] all calculation methods agree that the HOMO state of the ion-pair is due to the anion. [DEME][TFSI] is also the most electrochemically stable IL experimentally. [EMIM][TFSI] is electrochemically the least stable of the three ILs studied here and only for this ion-pair the hybrid DFT functionals predict that the HOMO state also involves the $\pi$-orbitals of the EMIM cation.

In the case of $\left[\mathrm{PYR}_{1,4}\right][\mathrm{TFSI}]$ vapor, the DOS calculated by the hybrid DFT functionals M06 and $\omega B 97 X-D$ agrees with the experimental UPS spectra better than the MP2 calculated DOS. This seems to validate the hybrid DFT calculation, which predicts that the HOMO of the $\left[\mathrm{PYR}_{1,4}\right][\mathrm{TFSI}]$ vapor is due to the TFSI anion. On the other hand, the aromatic cations have smaller experimental electrochemical stabilities and their HOMO should be due the cation, as predicted by the MP2 calculation. Therefore, the description of the electronic structure of the [PYR 1,4$]$ [TFSI] ion-pairs is not conclusive. As mentioned before, Lian et al. predicted that the $\mathrm{PYR}_{1,4}$ cation is more electrochemically stable than the EMIM cation [12].

It is also important to point out that the HOMO-LUMO gaps predicted by the hybrid M06 functional are reversed when compared to the experimental electrochemical stabilities. For example, the IL whose ion- 
pairs have the largest calculated HOMO-LUMO gap ([EMIM][TFSI]) is actually electrochemically the least stable. This seems to verify the claim that the HOMO-LUMO gaps from the calculation should not be blindly used to make conclusions about the anodic or cathodic stabilities.

Therefore, further study of the liquid phase electronic structure and the excited states of the ion-pairs of these ILs is necessary to make final conclusions.

\section{Acknowledgements}

The authors gratefully acknowledge the financial support by the Estonian Research Council (IUT2-25) and the Estonian Centre of Excellence in Research project "Advanced materials and high-technology devices for sustainable energetics, sensorics and nanoelectronics" TK141 (2014-2020.4.01.15-0011). The research leading to this result has been supported by the project CALIPSOplus under the Grant Agreement 730872 from the EU Framework Programme for Research and Innovation HORIZON 2020. The authors also wish to thank the staff of the MAX-lab for support during measurements. The authors commemorate early-deceased friend and colleague prof. Ergo Nõmmiste, who was one of the initiators and leaders of FinEstBeAMS consortium.

\section{References}

[1] R. Hayes, G. G. Warr, and R. Atkin, "Structure and Nanostructure in Ionic Liquids," Chemical Reviews, vol. 115, no. 13, pp. 6357-6426, JUL 82015 2015, doi: 10.1021/cr500411q.

[2] M. Earle et al., "The distillation and volatility of ionic liquids," Nature, vol. 439, no. 7078, pp. 831-834, FEB 162006 2006, doi: 10.1038/nature04451.

[3] M. P. S. Mousavi et al., "Ionic Liquids as Electrolytes for Electrochemical Double-Layer Capacitors: Structures that Optimize Specific Energy," Acs Applied Materials \& Interfaces, vol. 8, no. 5, pp. 3396-3406, FEB 102016 2016, doi: 10.1021/acsami.5b11353.

[4] C. Arbizzani, M. Biso, D. Cericola, M. Lazzari, F. Soavi, and M. Mastragostino, "Safe, highenergy supercapacitors based on solvent-free ionic liquid electrolytes," (in English), Journal of Power Sources, Article vol. 185, no. 2, pp. 1575-1579, Dec 2008, doi: 10.1016/j.jpowsour.2008.09.016.

[5] S. Zhang, S. Brahim, and S. Maat, "High-voltage operation of binder-free CNT supercapacitors using ionic liquid electrolytes," Journal of Materials Research, pp. 1-10, 2017.

[6] S. P. Ong, O. Andreussi, Y. B. Wu, N. Marzari, and G. Ceder, "Electrochemical Windows of Room-Temperature Ionic Liquids from Molecular Dynamics and Density Functional Theory Calculations," (in English), Chemistry of Materials, Article vol. 23, no. 11, pp. 2979-2986, Jun 2011, doi: 10.1021/cm200679y.

[7] M. P. S. Mousavi, S. Kashefolgheta, A. Stein, and P. Buhlmann, "Electrochemical Stability of Quaternary Ammonium Cations: An Experimental and Computational Study," (in English), Journal of the Electrochemical Society, Article vol. 163, no. 2, pp. H74-H80, 2016, doi: $10.1149 / 2.0671602$ jes. 
[8] Y. Zhang, C. Shi, J. Brennecke, and E. Maginn, "Refined Method for Predicting Electrochemical Windows of Ionic Liquids and Experimental Validation Studies," (in English), Journal of Physical Chemistry B, Article vol. 118, no. 23, pp. 6250-6255, JUN 12 2014 2014, doi: 10.1021/jp5034257.

[9] H. Matsumoto, H. Sakaebe, and K. Tatsumi, "Preparation of room temperature ionic liquids based on aliphatic onium cations and. asymmetric amide anions and their electrochemical properties as a lithium battery electrolyte," (in English), Journal of Power Sources, Article|Proceedings Paper vol. 146, no. 1-2, pp. 45-50, AUG 262005 2005, doi: 10.1016/j.jpowsour.2005.03.103.

[10] N. V. Ilawe, J. Fu, S. Ramanathan, B. M. Wong, and J. Z. Wu, "Chemical and Radiation Stability of Ionic Liquids: A Computational Screening Study," Journal of Physical Chemistry C, vol. 120, no. 49, pp. 27757-27767, Dec 2016, doi: 10.1021/acs.jpcc.6b08138.

[11] C. Lian, H. L. Liu, and J. Z. Wu, "Ionic Liquid Mixture Expands the Potential Window and Capacitance of a Supercapacitor in Tandem," Journal of Physical Chemistry C, vol. 122, no. 32, pp. 18304-18310, Aug 2018, doi: 10.1021/acs.jpcc.8b05148.

[12] C. Lian, H. L. Liu, C. Z. Li, and J. Z. Wu, "Hunting ionic liquids with large electrochemical potential windows," Aiche Journal, vol. 65, no. 2, pp. 804-810, Feb 2019, doi: 10.1002/aic.16467.

[13] P. Peljo and H. Girault, "Electrochemical potential window of battery electrolytes: the HOMO-LUMO misconception," (in English), Energy \& Environmental Science, Article vol. 11, no. 9, pp. 2306-2309, SEP 12018 2018, doi: 10.1039/c8ee01286e.

[14] V. Kisand et al., "Fragmentation and electronic decay of vacuum-ultraviolet-excited resonant states of molecular CsCl," Journal of Physics B-Atomic Molecular and Optical Physics, vol. 36, no. 19, 0953-4075, pp. 3909-3921, OCT 1420032003.

[15] A. Calo et al., "VUV excitation and electronic decay of rubidium halide molecules," Journal of Physics B-Atomic Molecular and Optical Physics, vol. 40, no. 12, 0953-4075, pp. 22612275, JUN 282007 2007, doi: 10.1088/0953-4075/40/12/004.

[16] A. Taylor, K. Lovelock, A. Deyko, P. Licence, and R. Jones, "High vacuum distillation of ionic liquids and separation of ionic liquid mixtures," (in English), Physical Chemistry Chemical Physics, Article vol. 12, no. 8, pp. 1772-1783, 2010 2010, doi: 10.1039/b920931j.

[17] I. Kuusik et al., "Valence band photoelectron spectra of [EMIM][BF4] ionic liquid vapor: Evidences of electronic relaxation," Journal of Molecular Liquids, vol. 223, pp. 939-942, NOV 2016 2016, doi: 10.1016/j.molliq.2016.08.114.

[18] I. Kuusik et al., "Valence electronic structure of [EMIM][BF4] ionic liquid: photoemission and DFT plus D study," Rsc Advances, vol. 8, no. 53, pp. 30298-30304, 2018 2018, doi: $10.1039 / \mathrm{c} 8 \mathrm{ra05865b}$.

[19] L. Reisberg et al., "UPS and DFT investigation of the electronic structure of gas-phase trimesic acid," Journal of Electron Spectroscopy and Related Phenomena, vol. 213, pp. 11-16, NOV 2016 2016, doi: 10.1016/j.elspec.2016.10.004.

[20] H. Yildirim, J. Haskins, C. Bauschlicher, and J. Lawson, "Decomposition of Ionic Liquids at Lithium Interfaces. 1. Ab Initio Molecular Dynamics Simulations," (in English), Journal of Physical Chemistry C, Article vol. 121, no. 51, pp. 28214-28234, DEC 282017 2017, doi: 10.1021/acs.jpcc.7b09657.

[21] J. Haskins, C. Bauschlicher, and J. Lawson, "Ab Initio Simulations and Electronic Structure of Lithium-Doped Ionic Liquids: Structure, Transport, and Electrochemical Stability," (in English), Journal of Physical Chemistry B, Article vol. 119, no. 46, pp. 14705-14719, NOV 192015 2015, doi: 10.1021/acs.jpcb.5b06951.

[22] J. Haskins, H. Yildirim, C. Bauschlicher, and J. Lawson, "Decomposition of Ionic Liquids at Lithium Interfaces. 2. Gas Phase Computations," (in English), Journal of Physical Chemistry C, Article vol. 121, no. 51, pp. 28235-28248, DEC 282017 2017, doi: 10.1021/acs.jpcc.7b09658.

[23] D. Strasser, F. Goulay, M. S. Kelkar, E. J. Maginn, and S. R. Leone, "Photoelectron spectrum of isolated ion-pairs in ionic liquid vapor," (in English), Journal of Physical Chemistry A, Article vol. 111, no. 17, pp. 3191-3195, May 2007, doi: 10.1021/jp0713231. 
[24] T. Kurisaki et al., "Surface Analysis of Ionic Liquids with and without Lithium Salt Using Xray Photoelectron Spectroscopy," (in English), Journal of Physical Chemistry B, Article vol. 116, no. 35, pp. 10870-10875, SEP 62012 2012, doi: 10.1021/jp301658k.

[25] T. Ikari, A. Keppler, M. Reinmöller, and B. W. J.D., "Surface Electronic Structure of Imidazolium-Based Ionic Liquids Studied by Electron Spectroscopy " e-Journal of Surface Science and Nanotechnology, vol. 8, pp. 241-245, 2010.

[26] O. Hofft, S. Bahr, M. Himmerlich, S. Krischok, J. Schaefer, and V. Kempter, "Electronic structure of the surface of the ionic liquid [EMIM][Tf2N] studied by metastable impact electron spectroscopy (MIES), UPS, and XPS," (in English), Langmuir, Article vol. 22, no. 17, pp. 7120-7123, AUG 152006 2006, doi: 10.1021/la060943v.

[27] A. Ulbrich, M. Reinmoller, W. J. D. Beenken, and S. Krischok, "Photoelectron spectroscopy on ionic liquid surfaces - Theory and experiment," (in English), Journal of Molecular Liquids, Article vol. 192, pp. 77-86, Apr 2014, doi: 10.1016/j.molliq.2014.01.007.

[28] S. Krischok et al., "A comparative study on the electronic structure of the 1-ethyl-3methylimidazolium bis(trifluoromethylsulfonyl)amide RT-ionic liquid by electron spectroscopy and first principles calculations," (in English), Zeitschrift Fur Physikalische Chemie-International Journal of Research in Physical Chemistry \& Chemical Physics, Article; Proceedings Paper vol. 220, no. 10-11, pp. 1407-1416, 2006, doi: 10.1524/zpch.2006.220.10.1407.

[29] M. Reinmoller et al., "Theoretical reconstruction and elementwise analysis of photoelectron spectra for imidazolium-based ionic liquids," (in English), Physical Chemistry Chemical Physics, Article vol. 13, no. 43, pp. 19526-19533, 2011, doi: 10.1039/c1cp22152c.

[30] R. Parna et al., "FinEstBeaMS - A wide-range Finnish-Estonian Beamline for Materials Science at the $1.5 \mathrm{GeV}$ storage ring at the MAX IV Laboratory," Nuclear Instruments \& Methods in Physics Research Section a-Accelerators Spectrometers Detectors and Associated Equipment, vol. 859, pp. 83-89, Jul 2017, doi: 10.1016/j.nima.2017.04.002.

[31] V. Pankratov et al., "Progress in development of a new luminescence setup at the FinEstBeAMS beamline of the MAX IV laboratory," (in English), Radiation Measurements, Article|Proceedings Paper vol. 121, pp. 91-98, FEB 2019 2019, doi: 10.1016/j.radmeas.2018.12.011.

[32] L. Minnhagen, "Spectrum and the energy levels of neutral argon, Ar i," Journal of the Optical Society of America, vol. 63, pp. 1185 - 1198, 1973.

[33] Y. Shao et al., "Advances in methods and algorithms in a modern quantum chemistry program package," (in English), Physical Chemistry Chemical Physics, Review vol. 8, no. 27, pp. 3172-3191, 2006 2006, doi: 10.1039/b517914a.

[34] J. Chai and M. Head-Gordon, "Long-range corrected hybrid density functionals with damped atom-atom dispersion corrections," (in English), Physical Chemistry Chemical Physics, Article vol. 10, no. 44, pp. 6615-6620, 2008 2008, doi: 10.1039/b810189b.

[35] A. Ikeda, Y. Nakao, H. Sato, and S. Sakaki, "Binding energy of transition-metal complexes with large pi-conjugate systems. Density functional theory vs post-hartree-fock methods," (in English), Journal of Physical Chemistry a, Article vol. 111, no. 30, pp. 7124-7132, AUG 2 2007 2007, doi: 10.1021/jp0708648.

[36] I. Lage-Estebanez, L. del Olmo, R. Lopez, and J. M. G. de la Vega, "The Role of Errors Related to DFT Methods in Calculations Involving Ion Pairs of Ionic Liquids," Journal of Computational Chemistry, vol. 38, no. 8, pp. 530-540, Mar 2017, doi: 10.1002/jcc.24707.

[37] I. Kuusik, M. Berholts, J. Kruusma, A. Tõnisoo, E. Lust, and V. Kisand, "Valence electronic structure of EMIM-B(CN)4: ion-pair vs bulk description," submitted to Chem. Phys. Lett., 2019.

[38] J. Cornil et al., "Valence electronic structure of pi-conjugated materials: Simulation of the ultraviolet photoelectron spectra with semiempirical Hartree-Fock approaches," (in English), Chemistry of Materials, Article vol. 11, no. 9, pp. 2436-2443, SEP 1999 1999, doi: $10.1021 / \mathrm{cm} 990117 \mathrm{q}$.

[39] N. Mardirossian and M. Head-Gordon, "Thirty years of density functional theory in computational chemistry: an overview and extensive assessment of 200 density functionals," 
(in English), Molecular Physics, Review vol. 115, no. 19, pp. 2315-2372, 2017 2017, doi: 10.1080/00268976.2017.1333614. 